\title{
Incomplete echocardiographic recovery at 6 months predicts long- term sequelae after intermediate-risk pulmonary embolism. A post- hoc analysis of the Pulmonary Embolism Thrombolysis (PEITHO) trial
}

\author{
Stefano Barco ${ }^{1}$. Mariaconcetta Russo ${ }^{1,2}$ - Eric Vicaut $^{3} \cdot$ Cecilia Becattini $^{4} \cdot$ Laurent Bertoletti $^{5,6,7}$. \\ Jan Beyer-Westendorf $f^{8,9} \cdot$ Hélène Bouvaist $^{10} \cdot$ Francis Couturaud $^{11} \cdot$ Thierry Danays $^{12} \cdot$ Claudia Dellas $^{13}$. \\ Daniel Duerschmied ${ }^{14} \cdot$ Klaus Empen $^{15} \cdot$ Emile Ferrari $^{16} \cdot$ Nazzareno Galiè $^{17}$. David Jiménez ${ }^{18}$. \\ Frederikus A. Klok ${ }^{1,19}$ - Maciej Kostrubiec ${ }^{20}$ - Matija Kozak ${ }^{21}$. Christian Kupatt ${ }^{22}$ - Irene M. Lang ${ }^{23}$. \\ Mareike Lankeit ${ }^{1,24,25}$. Nicolas Meneveau ${ }^{26,27}$. Massimiliano Palazzini ${ }^{17}$. Piotr Pruszczyk ${ }^{20}$. Matteo Rugolotto ${ }^{28}$. \\ Aldo Salvi ${ }^{29}$. Olivier Sanchez ${ }^{30,31,32}$. Sebastian Schellong ${ }^{33}$. Bozena Sobkowicz ${ }^{34}$. Guy Meyer ${ }^{30,31,35}$. \\ Stavros V. Konstantinides ${ }^{1,36}$
}

Received: 6 September 2018 / Accepted: 10 December 2018 / Published online: 18 December 2018

(c) The Author(s) 2018

\begin{abstract}
Introduction Symptoms and functional limitation are frequently reported by survivors of acute pulmonary embolism (PE). However, current guidelines provide no specific recommendations on which patients should be followed after acute PE, when follow-up should be performed, and which tests it should include. Definition and classification of late PE sequelae are evolving, and their predictors remain to be determined.

Methods In a post hoc analysis of the Pulmonary Embolism Thrombolysis (PEITHO) trial, we focused on 219 survivors of acute intermediate-risk PE with clinical and echocardiographic follow-up 6 months after randomisation as well as over the long term (median, 3 years after acute PE). The primary outcome was a composite of (1) confirmed chronic thromboembolic pulmonary hypertension (CTEPH) or (2) 'post-PE impairment' (PPEI), defined by echocardiographic findings indicating an intermediate or high probability of pulmonary hypertension along with New York Heart Association functional class II-IV. Results Confirmed CTEPH or PPEI occurred in 29 (13.2\%) patients, (6 with CTEPH and 23 with PPEI). A history of chronic heart failure at baseline and incomplete or absent recovery of echocardiographic parameters at 6 months predicted CTEPH or PPEI at long-term follow-up.

Conclusions CTEPH or PPEI occurs in almost one out of seven patients after acute intermediate-risk PE. Six-month echocardiographic follow-up may be useful for timely detection of late sequelae.
\end{abstract}

Keywords Chronic thromboembolic pulmonary hypertension $\cdot$ Post-PE impairment $\cdot$ Pulmonary embolism $\cdot$ Right ventricular dysfunction $\cdot$ Risk stratification

\section{Introduction}

Persisting symptoms and abnormalities of cardiorespiratory function or of echocardiographic parameters are frequently reported or detected after acute pulmonary embolism (PE).

Stefano Barco and Mariaconcetta Russo contributed equally to this study.

Stavros V. Konstantinides

stavros.konstantinides@unimedizin-mainz.de

Extended author information available on the last page of the article
They may be accompanied by reduced exercise capacity, impaired quality of life, and overall perception of a health status which is 'worse than before the acute PE event' [1-7]. The frequent clinical need for caring for these patients is not met by current guidelines, which provide no specific advice on whom, when, and how to follow after acute PE [8].

Recently, the concept of post-PE impairment (PPEI) or the 'post-PE syndrome' was proposed, encompassing various combinations of complaints and clinical findings as well as imaging, functional or haemodynamic abnormalities, at the far end of which stands a life-threatening obstructive vasculopathy, chronic thromboembolic pulmonary hypertension 
(CTEPH) [1, 4, 9-11]. CTEPH is often diagnosed with delay and the identification of predictors of CTEPH after an acute PE may help to reduce this delay. The definition of PPEI continues to evolve, and it is hoped that the results of ongoing studies will help to further optimise the detection, prediction, and classification of late PE sequelae [10].

In patients with intermediate-risk PE included in the Pulmonary Embolism Thrombolysis (PEITHO) study [12], for whom long-term follow-up was available [13], we analysed cardiopulmonary symptoms and abnormal echocardiographic parameters indicating pulmonary hypertension or right ventricular (RV) dysfunction 6 months after acute PE. Our aim was to find out whether these parameters were associated with CTEPH or PPEI at long-term follow-up. We thus sought to identify predictive tools for following the course of a patient who has suffered acute PE, allowing timely detection or exclusion of late clinical and haemodynamic sequelae.

\section{Patients and methods}

In PEITHO, a total of 1,006 patients with acute intermediate-risk PE were enrolled at 76 sites between November 2007 and July 2012 and randomised to receive tenecteplase or placebo (in addition to standard parenteral anticoagulation) [12]. Eligibility criteria included (1) an objectively confirmed diagnosis of acute PE with symptom onset 15 days or less before randomisation, (2) RV dysfunction detected on echocardiography or spiral computed tomography of the chest, and (3) a positive troponin I or T test [12]. The primary efficacy outcome was death or haemodynamic decompensation/collapse occurring within 7 days of randomisation.

An extension of the follow-up period to cover 2 years or longer was included in the third amendment of the study protocol, which was signed by 28 study sites having enrolled a total of 709 patients [13].

For the present analysis, we focused on PE survivors with available echocardiographic data at 6 months and over long-term follow-up ( 2 years or longer). Complete recovery of echocardiographic parameters between baseline and the 6-month visit was defined as normalisation of all echocardiographic parameters of RV dysfunction as documented by the investigators in the PEITHO case report form (Table 1). The New York Heart Association (NYHA) functional classification was used to provide an estimate of residual (or new) functional limitation during physical activity or at rest, and was assessed by the investigator team during a visit of the patient to the participating centre. The functional class prior to the acute PE event was not determined. The findings were collected in ad hoc-developed case report forms.

The outcome included a confirmed diagnosis of CTEPH or PPEI at long-term follow-up. The diagnostic workup for

Table 1 Definition of echocardiographic recovery at 6 months and of post-PE impairment at long-term follow-up

Recovery of echocardiographic parameters between baseline and 6 months

Echocardiographic parameters

a) $\mathrm{sPAP}>35 \mathrm{mmHg}$ (vs $\leq 35 \mathrm{mmHg}$ ) or tricuspid systolic velocity $>2.6 \mathrm{~m} / \mathrm{s}$ (vs $\leq 2.6 \mathrm{~m} / \mathrm{s}$ )

b) RVEDD $>30 \mathrm{~mm}$ (vs $\leq 30 \mathrm{~mm})$

c) $\mathrm{RVEDD} / \mathrm{LVEDD}>0.9$ (vs $\leq 0.9)$

d) Hypokinesia of the RV free wall

Complete recovery

Normalisation of all the echocardiographic parameters of right ventricular dysfunction listed above

Partial recovery

Normalisation of some, but not all, echocardiographic parameters

No recovery

Normalisation of none of the parameters that were elevated or abnormal at baseline

Combined study outcome

Confirmed diagnosis of CTEPH, or

Post-PE impairment (PPEI), defined as a combination of the following criteria [(a) and (b) both present)]:

Intermediate/high echocardiographic probability of pulmonary hypertension, * defined as estimated $\mathrm{sPAP}>35 \mathrm{~mm} \mathrm{Hg}$, or $\mathrm{sPAP} \leq 35 \mathrm{mmHg}$ associated with at least one of the following:

RVEDD > $30 \mathrm{~mm}$, or RVEDD/LVEDD > 0.9

hypokinesia of the RV free wall

Exertional dyspnoea of the NYHA class II, III or IV

CTEPH, chronic thromboembolic pulmonary hypertension; LVEDD, left ventricular end diastolic dimension; NYHA, New York Heart Association; PE, pulmonary embolism; RVEDD, right ventricular end diastolic dimension; RV, right ventricular; sPAP, systolic pulmonary artery pressure

*The definition of echocardiographic probability of pulmonary hypertension followed the criteria recommended by current European guidelines for standardising the follow-up assessment of patients with (chronic) pulmonary hypertension, but some of the parameters and cut-off values were adapted to correspond to the data collected in the case report forms of the PEITHO trial 
(suspected) CTEPH was performed at each participating site as part of standard medical care; it was not mandated by the PEITHO study protocol or its amendment concerning the extension of the follow-up period [13]. As shown in Table 1, PPEI was defined as echocardiographic findings indicating an intermediate or high probability of (chronic) pulmonary hypertension, combined with exertional dyspnoea of the New York Heart Association functional class II-IV. The definition of echocardiographic probability of pulmonary hypertension followed the criteria recommended by the current guidelines of the European Heart Association and European Respiratory Society [14], although some of the parameters and cut-off values had to be adapted to correspond to the data collected in the case report forms of the PEITHO trial (which had been defined before the pulmonary hypertension guidelines). CTEPH and PPEI cases were not independently adjudicated.

In the descriptive analysis of the patients' baseline characteristics and study outcomes for the overall population and for each treatment arm separately, percentages were used for categorical variables, and means (standard deviation, SD) or medians (interquartile range, IQR) for continuous variables. We analysed the effects of thrombolysis on the primary outcome by means of a two-sided Chi-square test for proportions. We searched for predictors of CTEPH or PPEI (the dependent variable) among clinically selected (1) baseline clinical characteristics, and (2) parameters assessed at 6-month assessment, notably NYHA functional class and an incomplete or absent recovery of echocardiographic parameters (vs complete recovery) compared to baseline by fitting univariate and multivariable stepwise logistic regression models. Missing values of single echocardiographic parameters were considered normal if $<5 \%$ or total. SAS software 9.2 was used for data analysis.

\section{Results}

Of 709 intermediate-risk patients randomised at the PEITHO sites which participated in the extension of the followup period, 136 patients died and 13 were lost during follow-up [13]. Among 560 survivors, echocardiography was performed in 219 patients at 6 months and over long-term follow-up (Fig. 1), of whom 112 were treated with tenecteplase and 107 with placebo. The median length of observation was 37 months (interquartile range, 27-49 months). In Table 2, the baseline parameters of survivors with echocardiographic follow-up data are compared with those without echocardiographic follow-up data.

At long-term follow-up, 29 of 219 (13.2\%) patients were diagnosed with CTEPH $(n=6)$ or fulfilled the PPEI criteria $(n=23)$. We did not detect any differences in the occurrence of CTEPH or PPEI among patients randomised to

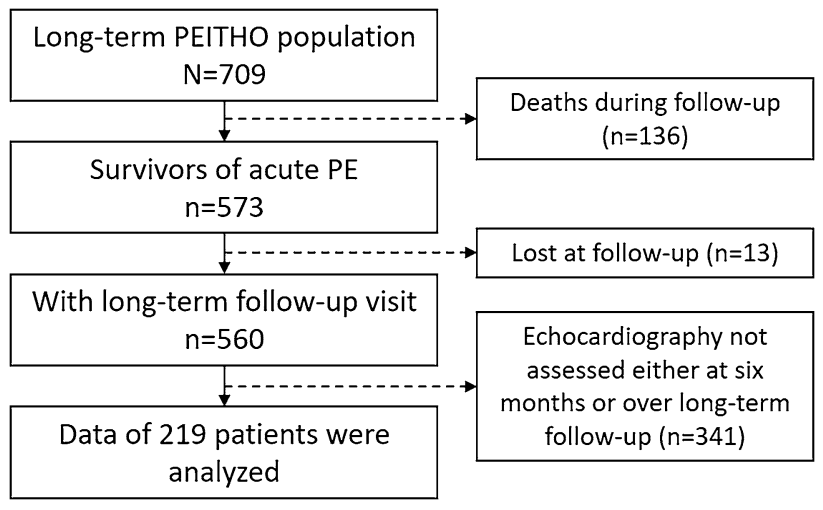

Fig. 1 Flowchart of patient inclusion for the present analysis

tenecteplase (14.3\%) versus anticoagulation alone (12.1\%) $(p=0.67)$.

Table 3 shows the unadjusted and adjusted estimates for the prediction of CTEPH or PPEI at long-term follow-up. Chronic heart failure, as reported by local study investigators, at baseline [adjusted odds ratio (OR) 7.72, 95\% confidence interval (CI) 1.28-46.65] and incomplete or absent recovery of echocardiographic parameters at 6 months [adjusted OR 7.14 (95\% CI 2.15-23.78)] were identified as independent predictors of CTEPH or PPEI.

\section{Discussion}

We investigated the long-term clinical and haemodynamic course of 219 survivors of acute intermediate-risk PE presenting with RV dysfunction and positive cardiac biomarkers, who had been enrolled in the PEITHO trial [12]. Longterm follow-up was conducted for a median of 37 months after acute PE. This examination revealed the presence of 'post-pulmonary embolism impairment' or CTEPH in $13.2 \%$ of the patients. The results of our regression analysis suggest that an abnormal follow-up echocardiogram, performed 'early' (6 months) after acute PE, may predict an elevated risk of persistent or progressive symptoms and RV dysfunction over the long term.

Our results indicate that persistence of clinical and haemodynamic impairment is a frequent complication after intermediate-risk PE and that 6-month clinical and echocardiographic assessment may be a useful tool for predicting these late PE sequelae. Consistent with what we had previously reported [13], we did not observe any impact of systemic thrombolysis on the risk of developing CTEPH or PPEI.

The definition of PPEI is still evolving; thus far, attention has been focused on the most severe but least frequent $\mathrm{PE}$ sequelae, CTEPH. In this regard, existing epidemiological data are characterised by a high degree of heterogeneity. A 
Table 2 Baseline characteristics of patients with versus those without complete echocardiographic assessment

\begin{tabular}{lcrr}
\hline & $\begin{array}{l}\text { Included } \\
(n=219)\end{array}$ & $\begin{array}{l}\text { Excluded } \\
(n=354)\end{array}$ & $p$ \\
\hline Age (years), mean (SD) & $64.8(14.5)$ & $64.6(16.7)$ & 0.90 \\
Male sex, $n(\%)$ & $111(50.7)$ & $157(44.4)$ & 0.14 \\
Body weight (kg), mean, (SD) & $84.0(15.7)$ & $83.0(18.4)$ & 0.50 \\
Systolic blood pressure (mmHg), mean (SD) & $133.6(17.2)$ & $130.5(18.1)$ & 0.04 \\
Heart rate (beats/min), mean (SD) & $91.3(17.4)$ & $93.8(16.5)$ & 0.08 \\
Respiratory rate (/min), mean (SD) & $21.6(5.6)$ & $21.4(5.5)$ & 0.72 \\
Oxygen administration, $n(\%)$ & $181(82.6)$ & $309(87.3)$ & 0.13 \\
Chronic obstructive pulmonary disease, $\mathrm{n}(\%)$ & $7(3.2)$ & $15(4.2)$ & 0.54 \\
Chronic heart failure, $n(\%)$ & $9(4.1)$ & $15(4.2)$ & 0.92 \\
Prior venous thromboembolism, $n(\%)$ & $51(23.3)$ & $98(27.7)$ & 0.24 \\
Active cancer, $n(\%)$ & $8(3.7)$ & $16(4.5)$ & 0.61 \\
Recent surgery or trauma, $n(\%)$ & $17(7.8)$ & $25(7.1)$ & 0.75 \\
Immobilisation, $n(\%)$ & $25(11.4)$ & $30(8.5)$ & 0.27 \\
Oestrogen use, $n(\%)$ & $14(6.4)$ & $27(7.6)$ & 0.58 \\
\hline
\end{tabular}

IQR, interquartile range; SD, standard deviation; NYHA, New York Heart Association

\begin{tabular}{lllll}
\hline & Unadjusted OR & $95 \%$ CI & Adjusted OR & 95\% CI \\
\hline Age $\leq 65$ years & 0.36 & $0.15-0.89$ & - & - \\
Male sex & 0.47 & $0.21-1.08$ & - & - \\
Chronic heart failure & 3.81 & $0.89-16.89$ & 7.72 & $1.28-46.65$ \\
Active cancer & 4.08 & $0.92-18.16$ & - & - \\
Prior venous thromboembolism & 1.10 & $0.44-2.76$ & - & - \\
Unprovoked pulmonary embolism & 0.99 & $0.37-2.61$ & - & - \\
Tenecteplase treatment & 1.19 & $0.54-2.62$ & - & - \\
NYHA II, III or IV (assessed at 6 months) & 3.20 & $1.33-7.71$ & - & - \\
Incomplete or absent recovery of echo & 4.77 & $1.80-12.63$ & 7.14 & $2.15-23.78$ \\
parameters (assessed at 6 months) & & & & \\
\hline
\end{tabular}

CI confidence interval, NYHA New York Heart Association, $O R$ odds ratio

\subsection{0}

0.14 0.50 0.04 0.08 0.72 0.13 0.54 0.92 0.24 0.61 0.75 0.27 0.58
Table 3 Factors associated with confirmed chronic thromboembolic pulmonary hypertension or post-pulmonary embolism impairment at longterm follow-up 
differences between the design, patient populations and outcomes in the ELOPE study and the PEITHO trial do not allow a direct comparison of the results of functional and echocardiographic assessment.

Our study has some limitations. First, we excluded patients with incomplete follow-up data; however, the comparison of baseline characteristics and 6-month findings between included and excluded patients (Table 2) suggests that selection bias is unlikely. Second, PEITHO did not include cardiopulmonary exercise or laboratory biomarker testing at follow-up, and thus the NYHA functional classification, a less standardised parameter [20], was used as the sole surrogate of functional impairment, combined with echocardiography at rest. Regarding the latter test, it needs to be mentioned that baseline and follow-up echocardiographic parameters were interpreted locally and not by a core laboratory. Third, a small overall number of patients were diagnosed with PPEI and particularly CTEPH, which led to large confidence intervals of the risk estimates limiting the ability to determine the strength of the association between the independent variables and the study outcome, and to adjust for important additional covariates. Finally, we cannot exclude the possibility that some patients may already have had CTEPH at the time of inclusion in PEITHO [21]. This represents a limitation of most existing studies in the field, as they were not designed to systematically search for pre-existing CTEPH using standardised criteria. Therefore, our findings might partly reflect a chronic condition present prior to the acute PE event, including pre-existing CTEPH or pulmonary hypertension of other cause(s), which may have contributed to the identification of 'chronic heart failure' as a significant predictor of outcome.

In light of the above limitations, and to the fact that no standardised CTEPH diagnosis protocol was mandated by the PEITHO trial protocol, no firm conclusions can be drawn from our analysis regarding the possible efficacy of systemic thrombolysis for prevention of late PE sequelae. Moreover, neither the PEITHO trial nor the present analysis was designed to directly address the question on which proportion of the patients with PPEI may ultimately progress to CTEPH, and at what rate this may happen.

In conclusion, we found that chronic thromboembolic pulmonary hypertension or the combination of exertional dyspnoea with persistent or progressing right ventricular dysfunction (termed post-pulmonary embolism impairment) occurs in almost one out of seven patients after acute intermediate-risk pulmonary embolism. Our results, which suggest that 6-month echocardiographic follow-up may be useful in detecting or predicting late sequelae of pulmonary embolism, must be confirmed by future, appropriately designed studies.
Funding This work was supported by the Federal Ministry of Education and Research (BMBF; 01KG0802, $01 \mathrm{EO} 1003$ and 01EO1503) in Germany; the Programme Hospitalier de Recherche Clinique (PHRC; AOM 03063, AOM 08231 and AOM 10171) in France; and a grant from the market authorisation holder of tenecteplase, Boehringer Ingelheim, to the trial sponsor, Assistance Publique Hôpitaux de Paris. The work of Stefano Barco, Frederikus Klok, Mareike Lankeit, and Stavros Konstantinides was supported by the German Federal Ministry of Education and Research (BMBF $01 \mathrm{EO} 1003$ and 01EO1503).

\section{Compliance with ethical standards}

Conflict of interest SB has received payment for travel accommodation/meeting expenses from Daiichi-Sankyo and Bayer HealthCare. MR has received congress and travel payments from NovoNordisk. DJ has served as an advisor or consultant for Bayer HealthCare Pharmaceuticals. FAK reports research grants from Bayer, Bristol-Myers Squibb, Boehringer Ingelheim, Daiichi-Sankyo, MSD and Actelion. OS has received payment for travel accommodation/meeting expenses from Bayer HealthCare, MSD, Actelion, Boehringer Ingelheim, Chiesi; board membership, consultancy and lecture honoraria to his institution from Actelion, Bayer HealthCare, Pfizer-BMS, and Chiesi; and institutional grants from Bayer HealthCare, Actelion, Daiichi-Sankyo, MSD and Portola. SVK reports having received consultancy and lecture honoraria from Bayer HealthCare, Boehringer Ingelheim, DaiichiSankyo, and Pfizer-Bristol-Myers Squibb; payment for travel accommodation/ meeting expenses from Bayer HealthCare; and institutional grants from Boehringer Ingelheim, Bayer HealthCare, Daiichi Sankyo, and MSD. Other authors: none reported.

Open Access This article is distributed under the terms of the Creative Commons Attribution 4.0 International License (http://creativeco mmons.org/licenses/by/4.0/), which permits unrestricted use, distribution, and reproduction in any medium, provided you give appropriate credit to the original author(s) and the source, provide a link to the Creative Commons license, and indicate if changes were made.

\section{References}

1. Klok FA, van der Hulle T, den Exter PL, Lankeit M, Huisman MV, Konstantinides S (2014) The post-PE syndrome: a new concept for chronic complications of pulmonary embolism. Blood Rev 28(6):221-226. https://doi.org/10.1016/j.blre.2014.07.003

2. Stevinson BG, Hernandez-Nino J, Rose G, Kline JA (2007) Echocardiographic and functional cardiopulmonary problems 6 months after first-time pulmonary embolism in previously healthy patients. Eur Heart J 28(20):2517-2524. https://doi.org/10.1093/ eurheartj/ehm295

3. Sharifi M, Bay C, Skrocki L, Rahimi F, Mehdipour M, Investigators M (2013) Moderate pulmonary embolism treated with thrombolysis (from the "MOPETT" Trial). Am J Cardiol 111(2):273277. https://doi.org/10.1016/j.amjcard.2012.09.027

4. Sista AK, Miller LE, Kahn SR, Kline JA (2017) Persistent right ventricular dysfunction, functional capacity limitation, exercise intolerance, and quality of life impairment following pulmonary embolism: systematic review with meta-analysis. Vasc Med 22(1):37-43. https://doi.org/10.1177/1358863X16670250

5. Klok FA, van Kralingen KW, van Dijk AP, Heyning FH, Vliegen HW, Kaptein AA, Huisman MV (2010) Quality of life in longterm survivors of acute pulmonary embolism. Chest 138(6):14321440. https://doi.org/10.1378/chest.09-2482 
6. Kline JA, Nordenholz KE, Courtney DM, Kabrhel C, Jones AE, Rondina MT, Diercks DB, Klinger JR, Hernandez J (2014) Treatment of submassive pulmonary embolism with tenecteplase or placebo: cardiopulmonary outcomes at 3 months: multicenter double-blind, placebo-controlled randomized trial. J Thromb Haemost 12(4):459-468. https://doi.org/10.1111/jth.12521

7. Kahn SR, Akaberi A, Granton JT, Anderson DR, Wells PS, Rodger MA, Solymoss S, Kovacs MJ, Rudski L, Shimony A, Dennie C, Rush C, Hernandez P, Aaron SD, Hirsch AM (2017) Quality of life, dyspnea, and functional exercise capacity following a first episode of pulmonary embolism: results of the ELOPE cohort study. Am J Med 130(8):990. https://doi.org/10.1016/j. amjmed.2017.03.033

8. Konstantinides SV, Torbicki A, Agnelli G, Danchin N, Fitzmaurice D, Galie N, Gibbs JS, Huisman MV, Humbert M, Kucher N, Lang I, Lankeit M, Lekakis J, Maack C, Mayer E, Meneveau N, Perrier A, Pruszczyk P, Rasmussen LH, Schindler TH, Svitil P, Vonk NA, Zamorano JL, Zompatori M (2014) 2014 ESC Guidelines on the diagnosis and management of acute pulmonary embolism: The Task Force for the Diagnosis and Management of Acute Pulmonary Embolism of the European Society of Cardiology (ESC)Endorsed by the European Respiratory Society (ERS). Eur Heart J 35(43):3033-3073. https://doi.org/10.1093/eurheartj/ ehu283

9. Kahn SR, Hirsch AM, Akaberi A, Hernandez P, Anderson DR, Wells PS, Rodger MA, Solymoss S, Kovacs MJ, Rudski L, Shimony A, Dennie C, Rush C, Geerts WH, Aaron SD, Granton JT (2017) Functional and exercise limitations after a first episode of pulmonary embolism: results of the ELOPE prospective cohort study. Chest 151(5):1058-1068. https://doi.org/10.1016/j.chest .2016 .11 .030

10. Konstantinides SV, Barco S, Rosenkranz S, Lankeit M, Held M, Gerhardt F, Bruch L, Ewert R, Faehling M, Freise J, Ghofrani HA, Grunig E, Halank M, Heydenreich N, Hoeper MM, Leuchte HH, Mayer E, Meyer FJ, Neurohr C, Opitz C, Pinto A, Seyfarth HJ, Wachter R, Zapf B, Wilkens H, Binder H, Wild PS (2016) Late outcomes after acute pulmonary embolism: rationale and design of FOCUS, a prospective observational multicenter cohort study. J Thromb Thrombolysis 42(4):600-609. https://doi.org/10.1007/ s11239-016-1415-7

11. Kramm T, Wilkens H, Fuge J, Schafers HJ, Guth S, Wiedenroth CB, Weingard B, Huscher D, Pittrow D, Cebotari S, Hoeper MM, Mayer E, Olsson KM (2018) Incidence and characteristics of chronic thromboembolic pulmonary hypertension in Germany. Clin Res Cardiol 107(7):548-553. https://doi.org/10.1007/s0039 2-018-1215-5

12. Meyer G, Vicaut E, Danays T, Agnelli G, Becattini C, BeyerWestendorf J, Bluhmki E, Bouvaist H, Brenner B, Couturaud F, Dellas C, Empen K, Franca A, Galie N, Geibel A, Goldhaber SZ, Jimenez D, Kozak M, Kupatt C, Kucher N, Lang IM, Lankeit M, Meneveau N, Pacouret G, Palazzini M, Petris A, Pruszczyk P, Rugolotto M, Salvi A, Schellong S, Sebbane M, Sobkowicz B, Stefanovic BS, Thiele H, Torbicki A, Verschuren F, Konstantinides SV, Investigators P (2014) Fibrinolysis for patients with intermediate-risk pulmonary embolism. N Engl J Med 370(15):1402-1411. https://doi.org/10.1056/NEJMoa1302097

13. Konstantinides SV, Vicaut E, Danays T, Becattini C, Bertoletti L, Beyer-Westendorf J, Bouvaist H, Couturaud F, Dellas C, Duerschmied D, Empen K, Ferrari E, Galie N, Jimenez D, Kostrubiec M, Kozak M, Kupatt C, Lang IM, Lankeit M, Meneveau N, Palazzini M, Pruszczyk P, Rugolotto M, Salvi A, Sanchez O,
Schellong S, Sobkowicz B, Meyer G (2017) Impact of thrombolytic therapy on the long-term outcome of intermediate-risk pulmonary embolism. J Am Coll Cardiol 69(12):1536-1544. https ://doi.org/10.1016/j.jacc.2016.12.039

14. Galie N, Humbert M, Vachiery JL, Gibbs S, Lang I, Torbicki A, Simonneau G, Peacock A, Vonk Noordegraaf A, Beghetti M, Ghofrani A, Gomez Sanchez MA, Hansmann G, Klepetko W, Lancellotti P, Matucci M, McDonagh T, Pierard LA, Trindade PT, Zompatori M, Hoeper M, Group ESCSD (2016) 2015 ESC/ERS Guidelines for the diagnosis and treatment of pulmonary hypertension: The Joint Task Force for the Diagnosis and Treatment of Pulmonary Hypertension of the European Society of Cardiology (ESC) and the European Respiratory Society (ERS): Endorsed by: Association for European Paediatric and Congenital Cardiology (AEPC), International Society for Heart and Lung Transplantation (ISHLT). Eur Heart J 37(1):67-119. https://doi.org/10.1093/eurhe artj/ehv317

15. Ende-Verhaar YM, Cannegieter SC, Vonk Noordegraaf A, Delcroix M, Pruszczyk P, Mairuhu AT, Huisman MV, Klok FA (2017) Incidence of chronic thromboembolic pulmonary hypertension after acute pulmonary embolism: a contemporary view of the published literature. Eur Respir J 49 (2). https://doi. org/10.1183/13993003.01792-2016

16. Pepke-Zaba J, Delcroix M, Lang I, Mayer E, Jansa P, Ambroz D, Treacy C, D’Armini AM, Morsolini M, Snijder R, Bresser P, Torbicki A, Kristensen B, Lewczuk J, Simkova I, Barbera JA, de Perrot M, Hoeper MM, Gaine S, Speich R, Gomez-Sanchez MA, Kovacs G, Hamid AM, Jais X, Simonneau G (2011) Chronic thromboembolic pulmonary hypertension $(\mathrm{CTEPH})$ : results from an international prospective registry. Circulation 124(18):19731981. https://doi.org/10.1161/CIRCULATIONAHA.110.015008

17. Delcroix M, Lang I, Pepke-Zaba J, Jansa P, D’Armini AM, Snijder R, Bresser P, Torbicki A, Mellemkjaer S, Lewczuk J, Simkova I, Barbera JA, de Perrot M, Hoeper MM, Gaine S, Speich R, Gomez-Sanchez MA, Kovacs G, Jais X, Ambroz D, Treacy C, Morsolini M, Jenkins D, Lindner J, Dartevelle P, Mayer E, Simonneau G (2016) Long-term outcome of patients with chronic thromboembolic pulmonary hypertension: results from an international prospective registry. Circulation 133(9):859-871. https ://doi.org/10.1161/CIRCULATIONAHA.115.016522

18. Ribeiro A, Lindmarker P, Johnsson H, Juhlin-Dannfelt A, Jorfeldt L (1999) Pulmonary embolism: one-year follow-up with echocardiography doppler and five-year survival analysis. Circulation 99(10):1325-1330

19. Tapson VF, Platt DM, Xia F, Teal SA, de la Orden M, Divers CH, Satler CA, Joish VN, Channick RN (2016) monitoring for pulmonary hypertension following pulmonary embolism: the INFORM study. Am J Med 129(9):978-985 e972. https://doi.org/10.1016/j. amjmed.2016.03.006

20. Raphael C, Briscoe C, Davies J, Ian Whinnett Z, Manisty C, Sutton R, Mayet J, Francis DP (2007) Limitations of the New York Heart Association functional classification system and self-reported walking distances in chronic heart failure. Heart 93(4):476-482. https://doi.org/10.1136/hrt.2006.089656

21. Guerin L, Couturaud F, Parent F, Revel MP, Gillaizeau F, Planquette B, Pontal D, Guegan M, Simonneau G, Meyer G, Sanchez O (2014) Prevalence of chronic thromboembolic pulmonary hypertension after acute pulmonary embolism. Prevalence of CTEPH after pulmonary embolism. Thromb Haemost 112(3):598-605. https://doi.org/10.1160/TH13-07-0538 


\section{Affiliations}

Stefano Barco ${ }^{1}$ - Mariaconcetta Russo ${ }^{1,2}$ - Eric Vicaut ${ }^{3} \cdot$ Cecilia Becattini $^{4} \cdot$ Laurent Bertoletti $^{5,6,7}$. Jan Beyer-Westendorf $f^{8,9} \cdot$ Hélène Bouvaist $^{10} \cdot$ Francis Couturaud $^{11} \cdot$ Thierry Danays $^{12} \cdot$ Claudia Dellas $^{13}$. Daniel Duerschmied ${ }^{14} \cdot$ Klaus Empen $^{15} \cdot$ Emile Ferrari $^{16} \cdot$ Nazzareno Galiè $^{17}$. David Jiménez ${ }^{18}$. Frederikus A. Klok,19 . Maciej Kostrubiec ${ }^{20}$ - Matija Kozak ${ }^{21}$. Christian Kupatt ${ }^{22}$ • Irene M. Lang ${ }^{23}$. Mareike Lankeit ${ }^{1,24,25}$. Nicolas Meneveau ${ }^{26,27}$. Massimiliano Palazzini ${ }^{17}$. Piotr Pruszczyk ${ }^{20}$. Matteo Rugolotto ${ }^{28}$.

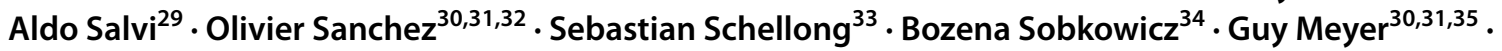
Stavros V. Konstantinides ${ }^{1,36}$

1 Center for Thrombosis and Hemostasis, University Medical Center, Langenbeckstrasse 1, Mainz, Germany

2 Department of Internal Medicine, University of Pavia, Pavia, Italy

3 Clinical Research Unit, Assistance Publique Hôpitaux de Paris, Fernand-Widal Hospital, University Paris Diderot, Paris, France

4 Department of Internal and Cardiovascular Medicine-Stroke Unit, University of Perugia, Perugia, Italy

5 Department of Vascular Medicine and Therapy, Saint-Etienne University Hospital Center, Saint-Etienne, France

6 INSERM (National Institute of Health and Medical Research) U1059, Saint-Etienne, France

7 INSERM CIC1408, Saint-Etienne, France

8 Department of Medicine, Division of Hematology, Thrombosis Research, University Hospital Carl Gustav Carus, Technical University Dresden, Dresden, Germany

9 King's Thrombosis Service, Department of Hematology, King's College London, London, UK

10 Cardiology Service, Michallon Hospital, Grenoble University Hospital Center, Grenoble, France

11 Department of Internal Medicine and Pulmonology, La Cavale Blanche Hospital, CIC INSERM 1412, University of Western Brittany, E 3878 (GETBO), Brest, France

12 Boehringer Ingelheim, Reims, France

13 Department of Paediatric Cardiology and Intensive Care, University Medical Center Göttingen, Göttingen, Germany

14 Heart Center, Faculty of Medicine, University of Freiburg, Freiburg, Germany

15 Ernst Moritz Arndt Greifswald University Hospital, Greifswald, Germany

16 Department of Cardiology, University Hospital of Nice, Nice, France

17 Department of Experimental, Diagnostic and Specialty Medicine-DIMES, Bologna University Hospital, Bologna, Italy

18 Department of Respiratory Diseases, Ramon y Cajal Hospital, IRYCIS, Madrid, Spain
19 Department of Thrombosis and Hemostasis, Leiden University Medical Center, Leiden, The Netherlands

20 Department of Internal Medicine and Cardiology, Medical University of Warsaw, Warsaw, Poland

21 University Medical Center, Ljubljana, Slovenia

22 Klinikum Rechts der Isar, TU Munich, and German Center for Cardiovascular Research (DZHK), Partner Site Munich Heart Alliance, Munich, Germany

23 Department of Cardiology, Vienna General Hospital, Medical University of Vienna, Vienna, Austria

24 Department of Internal Medicine and Cardiology, Campus Virchow Klinikum (CVK), Charité-University Medicine Berlin, Berlin, Germany

25 Cardiology and Pulmonology Clinic, University Medical Center Göttingen, Göttingen, Germany

26 Department of Cardiology, Equipe d'Accueil 3920, Structure Fédérative de Recherche, University Hospital Jean Minjoz, Besançon, France

27 F-CRIN INNOVTE, St-Etienne, France

28 Department of Cardiology, Ca'Foncello Hospital, Treviso, Italy

29 Azienda Ospedaliero-Universitaria Ospedali Riuniti di Ancona, Ancona, Italy

30 Université Paris Descartes, Sorbonne Paris Cité, Paris, France

31 Pulmonology and Intensive Care Service, Georges Pompidou European Hospital, Assistance Publique Hôpitaux de Paris, Paris, France

32 INSERM UMR S 1140, Paris, France

33 Municipal Hospital of Dresden-Friedrichstadt, Dresden, Germany

34 Medical University, Bialystok, Poland

35 INSERM UMR S 970, Paris, France

36 Democritus University of Thrace, Alexandroupolis, Greece 